\title{
Quantum dynamics and delocalization in coherently driven one-dimensional double-well system
}

\author{
Akira Igarash $*$ \\ Graduate School of Science and Technology, Niigata University, \\ Ikarashi 2-Nochou 8050, Niigata 950-2181, Japan \\ Hiroaki S. Yamaddit \\ YPRL, 5-7-14 Aoyama, Niigata 950-2002, Japan
}

(Dated: November 20, 2018)

\begin{abstract}
We numerically study influence of a polychromatic perturbation on wave packet dynamics in onedimensional double-well potential. It is found that time-dependence of the transition probability between the wells shows two kinds of the motion typically, coherent oscillation and irregular fluctuation combined to the delocalization of the wave packet, depending on the perturbation parameters. The coherent motion changes the irregular one as the strength and/or the number of frequency components of the perturbation increases. We discuss a relation between our model and decoherence in comparing with the result under stochastic perturbation. Furthermore we compare the quantum fluctuation, tunneling in the quantum dynamics with ones in the semiquantal dynamics.
\end{abstract}

PACS numbers: 05.45.-a, 03.65.-w

\section{INTRODUCTION}

Quantum fluctuation can suppress chaotic motion of wave packet in the phase space due to the quantum interference, as seen in kicked rotor [1, 2]. On the contrary, the quantum fluctuation can enhance the chaotic motion of wave packet due to tunneling effect as seen in kicked double-well model [3, 4]. The relation between chaotic behavior and tunneling phenomenon in classically chaotic systems is interesting and important subject in study of quantum physics [5, 6]. Recently, the semiclassical description for the tunneling phenomena in a classically chaotic system have been developed by several groups [7, 8, 9$]$.

Lin and Ballentine studied interplay between the tunneling and classical chaos for a particle in a double-well potential with oscillatory driving force [10]. They found that coherent tunneling takes place between small isolated classical stable regions of phase space bounded by Kolmogorov-Arnold-Moser (KAM) surfaces, which are much smaller than the volume of a single potential well.

Hänggi and the coworkers studied the chaos-suppressed tunneling in the driven double-well model in terms of the Floquet formalism [11]. They found a one-dimensional manifold in the parameter space, where the tunneling completely suppressed by the coherent driving. The time-scale for the tunneling between the wells diverges because of intersection of the ground state doublet of the quasienergies.

While the mutual influence of quantum coherence and classical chaos has been under investigation since many years ago, the additional effects caused by coupling the

\footnotetext{
*Electronic address: f99j806b@mail.cc.niigata-u.ac.jp

${ }^{\dagger}$ Electronic address: hyamada@uranus.dti.ne.jp
}

chaotic system to the other degrees of freedom (DOF) or an environment, namely decoherence and dissipation, have been studied only rarely 11, 12] as well as the tunneling phenomena in the chaotic system. Since mideighties there are some studies on environment-induced quantum decoherence by coupling the quantum system to a reservoir [13, 14, 15]. Recently quantum dissipation due to the interaction with chaotic DOF has been also studied 16, 17, 18].

In this paper we numerically investigate the relation quantum fluctuation, tunneling and decoherence combined to the delocalization in wave packet dynamics in one-dimensional double-well system driven by polychromatic external field.

Before closing this section, we refer to a study on a delocalization phenomenon by a perturbation with some frequency components in the other model. Casati et al. have reported that the kicked rotator model with a frequency modulation amplitude of kick can be mapped to the tight-binding form (Loyld model) on higherdimensional lattice in solid-state physics under very specific condition [19, 20]. Then the number $M$ of the incommensurate frequencies corresponds the dimensionality of the tight-binding system. The problem can be efficiently reduced to a localization problem in $M+1$ dimension. As seen in the case of kicked rotators, we can also expect that in the double-well system the coupling with oscillatory perturbation is roughly equivalent to an increase in effective degrees of freedom and a transition from a localized wave packet to delocalized one is enhanced by the polychromatic perturbation. The concrete confirmation of the naive expectation is one of aims of this numerical work.

We present the model in the next section. In Sect.3, we show the details of the numerical results of the timedependence of the transition probability between the wells based on the quantum dynamics. Section 4 contains 
the summary and discussion. Furthermore, in appendix $\mathrm{A}$, we gave details of the classical phase space portraits in the polychromatically perturbed double-well system and some considerations to the effect of polychromatic perturbation. In appendix B, a simple explanation for the perturbed instanton tunneling picture is given.

\section{MODEL}

We consider a system described by the following Hamiltonian,

$$
\begin{aligned}
& H(t)=\frac{p^{2}}{2}+\frac{q^{4}}{4}-A(t) \frac{q^{2}}{2}, \\
& A(t)=a-\frac{1}{\sqrt{ } M} \sum_{i=1}^{M} \epsilon_{i} \sin \left(\Omega_{i} t+\theta_{i}\right) .
\end{aligned}
$$

For the sake of simplicity, $\left\{\epsilon_{i}\right\}$ and $\left\{\theta_{i}\right\}$ are taken as $\epsilon_{i}=\epsilon, \theta_{i}=0, i=1,2, . ., M$ in the present paper. Then $M$ is the number of frequency components of the external field and $\epsilon$ is the perturbation strength respectively. $\left\{\Omega_{i}\right\}$ are order of unity and mutually incommensurate frequencies. We choose off-resonant frequencies which are far from both classical and quantum resonance in the corresponding unperturbed problem. The parameter $a$ adjusts the distance between the wells and we set $a=5$ to make some energy doublets below the potential barrier. Note that Lin et al. dealt with a double-well system driven by forced oscillator (Duffing-like model), therefore, the asymmetry of the potential plays an important role in the chaotic behavior and tunneling transition between the symmetry-related KAM tori [10, 11]. However, in our model the potential is remained symmetric during the time evolution process, and different mechanism from the forced oscillation makes the classical chaotic behavior 21, 22, 23].

In the previous paper 24] we presented numerical results concerning a classical and quantum description of the field-induced barrier tunneling under the monochromatic perturbation $(M=1)$. In the unperturbed doublewell system $(M=0)$ the instanton describes the coherent tunneling motion of the initially localized wave packet. It is also shown that the monochromatic perturbation can breaks the coherent motion as the perturbation strength increases near the resonant frequency in the previous paper. In the classical dynamics of our model, outstanding feature different from previous studies is parametric instability caused by the polychromatic perturbation.

Based on our criterion given below, we roughly estimate the type of the motion, i.e. the coherent and irregular motions, in a regime of the parameter space spanned by the amplitude and the number of frequency components of the oscillatory driving force. It is suggested that the occurrence of the irregular motion is related to dissipative property which is organized in the quantum physics [25]. The classical phase space portraits and sim- ple explanation of relation to the dissipative property are given in appendix A.

\section{NUMERICAL RESULTS}

We use Gaussian wavepacket with zero momentum as the initial state, which is localized in the right well of the potential.

$$
\psi(q, t=0)=(\sigma \pi)^{1 / 4} \exp \left\{-\frac{\left(q-q_{0}\right)^{2}}{2 \sigma}\right\},
$$

where $q_{0}=\sqrt{ } a \simeq 2.236$ means a bottom of the right well. The Gaussian wavepacket can be approximately generated by the linear combination of the ground state doublet as $\psi(q, t=0) \simeq \frac{1}{\sqrt{ } 2}\left(\varphi_{0}(q)+\varphi_{1}(q)\right)$, where $\varphi_{0}$ and $\varphi_{1}$ denote the ground state doublet. The recurrence time for the wavepacket is $T \equiv 2 \pi \hbar / \Delta \epsilon_{01} \sim 9.4 \times 10^{3}$ in the unperturbed case $(M=0)$, where $\Delta \epsilon_{10}$ is the energy difference between the tunneling doublet of the ground state. We set the spread of the initial packet $\sigma=$ $1 / 3.4(\sim 0.3)$ and $\hbar=1.0$ for simplicity throughout this paper. Indeed, the ammonia molecule is well described by two doublets below the barrier heigth in unperturbed case.

We numerically calculate the solution $\psi(q, t)$ of timedependent Schrödinger equation by using second order unitary integration with time step $\delta \sim 10^{-2}$. We define transition probability of finding the wave packet in the left well,

$$
P_{L}(t) \equiv \int_{-\infty}^{0}|\psi(q, t)|^{2} d q
$$

In the cases that the perturbation strength is relatively small, $P_{L}(t)$ can be interpreted as the tunneling probability that the initially localized wave packet goes through the central energy barrier and reaches the left well. We can expect that the transition probability $P_{L}(t)$ is enhanced as the number $M$ of the frequency components increases up to some extent because of the increasing of the stochasticity in the total system.

Figure 1 shows the time-dependence of $P_{L}(t)$ for various combinations of $\epsilon$ and $M$. Apparently we can observe the coherent and irregular motions. The coherent motion of the wave packet can be well-described by the semiquantal picture in a sense that the wave packet does not delocalize to the fully delocalized state. The semiquantal picture decomposes the motion of the wave packet into evolution of the centroid motion and the spreading and squeezing of the packet [3]. (See Subsect.3.5.) For example, in cases of relatively small perturbation strength $(\epsilon=0.4)$, coherent motion remains still up to relatively large $M(=5)$.

It is important to emphasize that the tunneling contribution to the transition probability $P_{L}$ is not so significant for large $\epsilon$ and/or $M$. Then $P_{L}$ may be interpreted 

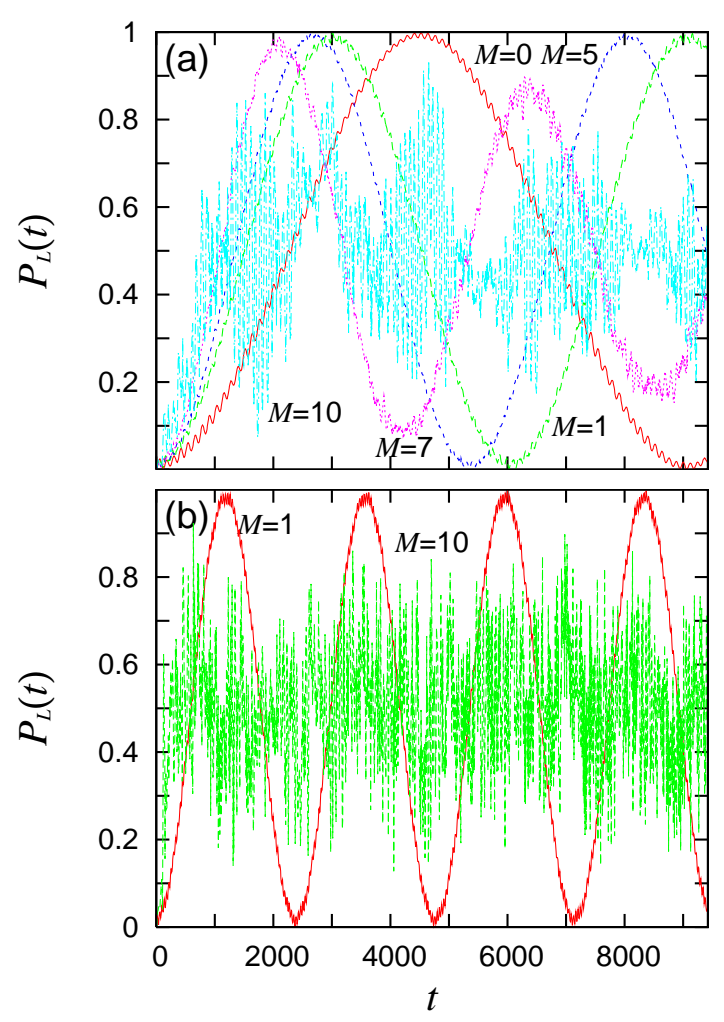

FIG. 1: (Color online) Tunneling probability $P_{L}(t)$ as a function of time $t$ for various $M$ 's. (a) $\epsilon=0.4$. (b) $\epsilon=0.8$. The calculation time is same order to Heisenberg time in the unperturbed case.

as a barrier crossing probability due to the activationtransition because the energy of wave packet increases over the barrier height in the parameter range. Especially, in the relatively large perturbation regime we can interpret the delocalized states as chaos-induced delocalization in a sense that the classical chaos enhances the quantum barrier crossing rate quite significantly. The chaotic behavior in the classical dynamics is given in appendix A, based on the classical Poincaré section and so on [26]. In the present section, we mainly focus on the transition of the quantum state from the localized wavepacket to delocalized state based on the data of numerical calculation.

\section{A. decoherence of the dynamics}

Once the wave packet incoherently spreads into the space as the $M$ and/or $\epsilon$ increase, the wavepacket is delocalized and never return to Gaussian shape again within the numerically accessible time. Apparently, we regard the delocalized quantum state as a decoherent state in a sense that the behavior of the wave packet is similar to that of the stochastically perturbed case.
(See Fig.5(a).) In case of relatively small perturbation strength $(\epsilon=0.4)$, the decoherence of quantum dynamics appears at around $M \simeq 7$, and $P_{L}(t)$ fluctuates irregularly in case of large $M(=10)$. In short, the irreversible delocalization of a Gaussian wave packet generates a transition from coherent oscillation to irregular fluctuation of $P_{L}(t)$. We have confirmed that the similar behavior is also observed for other sets of values of the frequencies and the different initial phases $\left\{\theta_{i}\right\}$ of the perturbation.

Here, we define a degree of coherence $\Delta P_{L}$ of the timedependence of $P_{L}(t)$, based on the fluctuation of the transition probability in order to estimate quantitatively the difference between coherent and incoherent motions.

$$
\Delta P_{L} \equiv \sqrt{ }\left\langle\left(P_{L}(t)-\left\langle P_{L}(t)\right\rangle_{T}\right)^{2}\right\rangle_{T},
$$

where $\langle\ldots\rangle_{T}$ represents time average value for a period $T=9.4 \times 10^{3}$. Note that we used $\Delta P_{L}$ in order to express the decoherence of the tunneling osccilation of the transition probability in the parametrically perturbed doublewell system. On the other hand, the other quantities such as purity, linear entropy and fidelity, are sometimes used to characterize the decoherence of the quantum system [27. The transition of the dynamical behavior based on the fidelity for description of the decoherence in the double-well system will be given elsewhere [26].

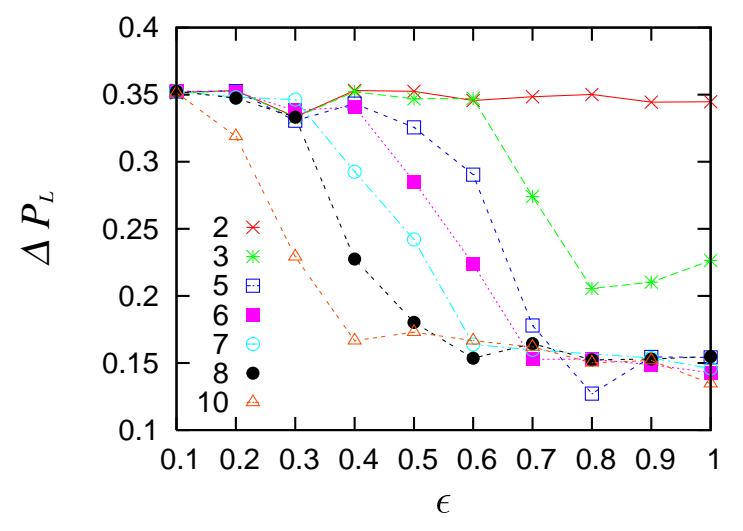

FIG. 2: Perturbation strength $\epsilon$ dependence of the degree of coherence $\Delta P_{L}$ of tunneling probability for various $M$ 's. The $\Delta P_{L}$ is numerically estimated by $P_{L}(t)$.

Figure 2 shows the perturbation strength dependence of $\Delta P_{L}$ for various $M$ 's. We roughly divide the type of motion of wave packet into three ones as follows. In the coherent motions, the value of $\Delta P_{L}$ 's is almost same to the unperturbed case, i.e. $\Delta P_{L} \geq 0.3$, in which cases the instanton-like picture is valid [28]. A simple explanation of the perturbed instanton is given in appendix B.

In the irregular motions which are similar to the stochastically perturbed case, the value of $\Delta P_{L}$ 's becomes much smaller, i.e. $\Delta P_{L} \lesssim 0.2$. As a matter of course, there are the intermediate cases between the coherent and the irregular motions, $0.2 \lesssim \Delta P_{L} \lesssim 0.3$. Note that the exact criterion of the intermediate motion is not 
important in the present paper because we can expect that the transitional cases approach to the irregular case in the long-time behavior. It should be stressed that the critical value $\epsilon_{c}$ exists, which divides the behavior of $P_{L}(t)$ into regular and irregular motions.

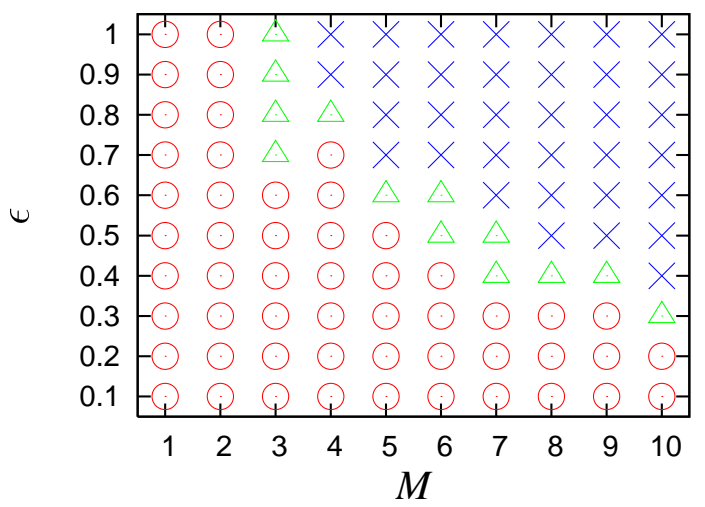

FIG. 3: (Color online) Approximate phase diagram in the parameter space spanned by the amplitude and the number of frequency components of the oscillatory driving force. The motions are plotted as different marks based on the degree of coherence $\Delta P_{L}$. Circles $(\bigcirc), \operatorname{crosses}(\times)$, and triangles $(\triangle)$ denote coherent motions $\left(\Delta P_{L} \geq 0.3\right)$, irregular motions $\left(\Delta P_{L} \leq 0.2\right)$, and the transitional cases $\left(0.2 \leq \Delta P_{L} \leq 0.3\right)$ respectively.

Figure 3 shows a classification of the motion in the parameter space which is estimated by the value of the degree of coherence $\Delta P_{L}$. It seems that two kinds of the motion, i.e. coherent and irregular motions, are divided by the thin layer corresponding to the "transitional case". As $M$ increases, decoherence of the motion appears even for small $\epsilon$. The numerical estimation suggests that there are the critical values $\epsilon_{c}(M)$ of the perturbation strength depending on $M$. When the perturbation strength $\epsilon$ exceeds the critical value $\epsilon_{c}(M)$ for some $M$, the tunneling oscillation loses the coherence. The approximated phase diagram roughly same as the diagram generated by maximal Lyapunov exponent of the classical dynamics. (See appendix A.)

\section{B. reduction of the tunneling period for $\epsilon<\epsilon_{c}$}

In this subsection we give a consideration to the reduction of the tunneling period in the regular motion regime $\epsilon<\epsilon_{c}$.

Figure 4 shows the $\epsilon$-dependence of the the period $T_{\text {tun }}$ of the tunneling oscillation estimated by the numerical data $P_{L}(t)$ in the coherent motion regime $\epsilon<\epsilon_{c}$. We can observe the monotonically decreasing of the tunneling period as the perturbation strength increases. In the monochromatically perturbed case, the reduction of the tunneling period can be interpreted by applying the Floquet theorem to the quasi-energy states and the quasienergy as the Hamiltonian is time-periodic $H(t+2 \pi / \Omega)=$

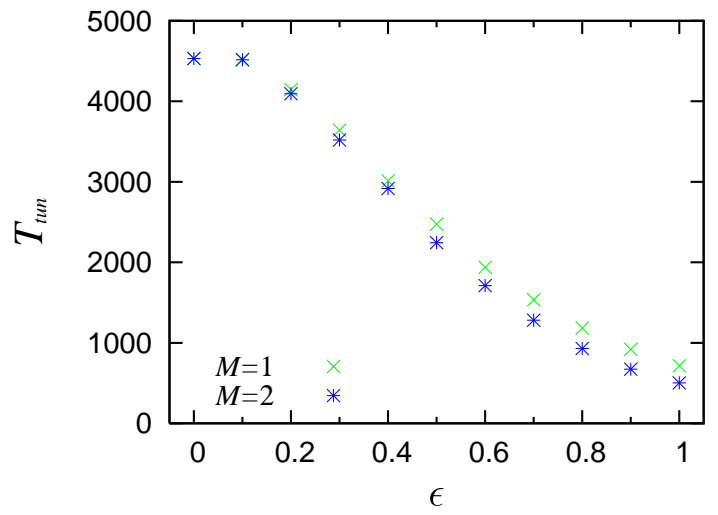

FIG. 4: Perturbation strength dependence of tunneling pe$\operatorname{riod} T_{\text {tun }}$ for $M=1$ and $M=2$ in the coherent motion regime $\epsilon<\epsilon_{c}$.

$H(t)$. When the wave packet does not effecively absorb the energy from the external perturbation the timedependence of the quantum state can be described by the linear combination of a doublet of quasi-degenerate ground states with opposite parity because we prepare the initial state in $\psi(q, t=0) \simeq \frac{1}{\sqrt{ } 2}\left(\varphi_{0}(q)+\varphi_{1}(q)\right)$ and the evolution is adiabatic. In the two-state approximation that the avoided crossing of the eigenvalues dynamics does not appear during the time evolution, it is expected that the state evolves as,

$\psi(q, t) \simeq \frac{1}{\sqrt{ } 2}\left(u_{0}(q, t) e^{-i \int_{0}^{t} E_{0}(\tau) d \tau}+u_{1}(q, t) e^{-i \int_{0}^{t} E_{1}(\tau) d \tau}\right)$

where $\left(E_{0}(t), E_{1}(t)\right)$ and $\left(u_{0}(t), u_{1}(t)\right)$ denote the quasienergies and Floquet states of the time-periodic Hamiltonian [29]. Under the approximation we expect the following relation,

$$
T_{\text {tun }} \simeq \frac{\pi}{\Delta E_{01}},
$$

where $\Delta E_{01}$ means quasi-energy splitting of the ground state doublet due to the tunneling between the wells.

Let us confirm the relation in Eq.(7) numerically. In Fig. 5 we show the $\epsilon-$ dependence of $\pi / \Delta E_{01}$. The behavior is analogus to the $\epsilon$-dependence of the tunneling period of the oscillation $P_{L}(t)$ in Fig. 4, in the weak perturbation regime.

The similar correspondence between the tunneling period and the change of the quasi-energy splitting have been reported for the other double-well system by Tomsovic et al. 30, 31, 32, 33. It is well-known that the chaos around the separatrix contributes to the enhancement of the tunneling split between the doublet, i.e. chaosassisted tunneling. The reduction of the tunneling period can be approximately explained by the chaos-assisted instanton picture in the coherent oscilation regime $\epsilon<\epsilon_{c}$. The simple explanation for the perturbed instanton picture based on the width of the chaotic layer in the classical dynamics is given in appendix B. (See also appendix A.) 


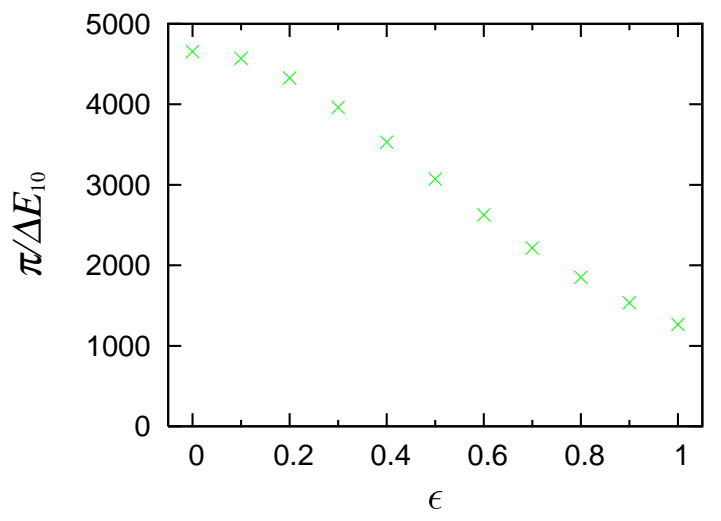

FIG. 5: Perturbation strength dependence of quasi-energy splitting in the coherent motion regime $\epsilon<\epsilon_{c}$ in the monochromatically perturbed case $(M=1)$.

Generally speaking, as the number of frequencies $M$ increases the tunneling period is more reduced as seen in Fig.4 although we do not have analytic representation in the polychromatically perturbed cases. We conjecture that as seen in appendix A the increasing of the width of the stochastic layer contributes the reduction of the tunneling period even in the polychromatically perturbed cases.
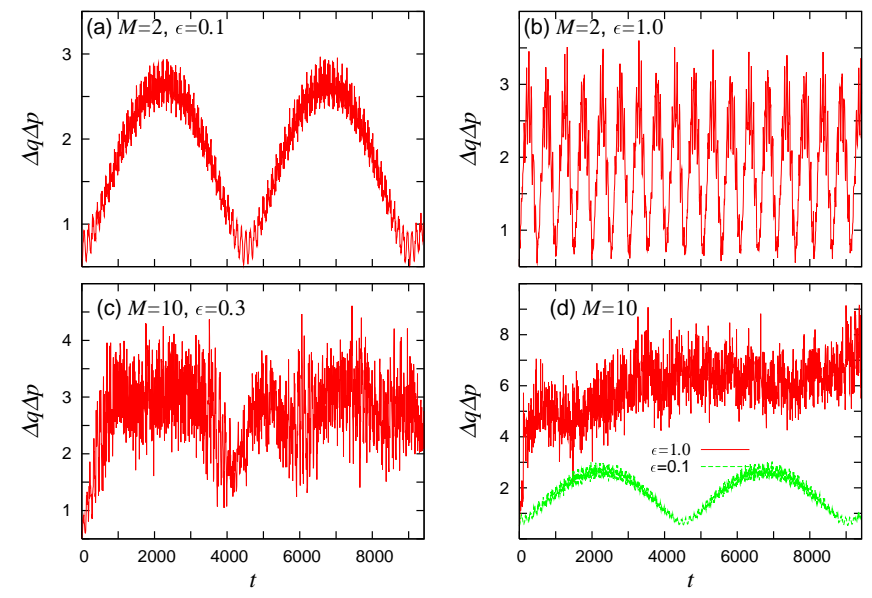

FIG. 6: Plots of the uncertainty product $\Delta q \Delta p$ as a function of time for some combinations of the parameters. (a) $M=2$, $\epsilon=0.1$. (b) $M=2, \epsilon=1.0$. (c) $M=10, \epsilon=0.3$. (d) $M=10, \epsilon=1.0$.

\section{C. uncertainly product}

Here let us investigate the spread of the wave packet in the phase space $(q, p)$. Hitherto we mainly investigated the dynamics in $q$-space by $P_{L}(t)$. The phase space volume gives a part of the compensating information for the phase space dynamics of the wave packet. Figure 6
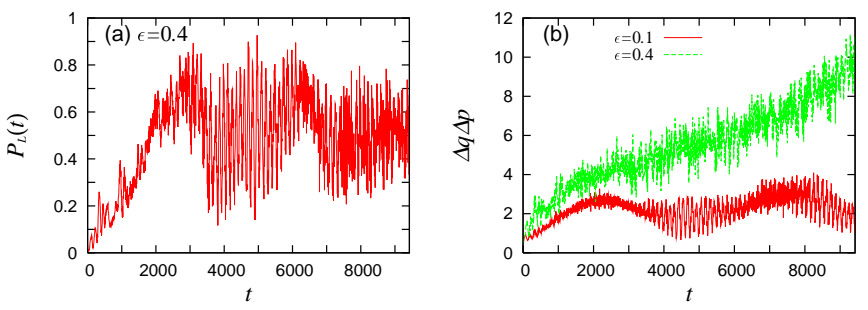

FIG. 7: (a)Tunneling probability $P_{L}(t)$ as a function of time $t$ under stochastic perturbation with $\epsilon=0.4$. (b)Plots of the uncertainty product $\Delta q \Delta p$ versus time for various $\epsilon^{\prime}$ s with the stochastic perturbation. The stochastic perturbation strength $\epsilon$ is normalized to be equivalent to one of the polychromatic perturbation.

presents the uncertainly product, i.e. phase space volume, as a function of time for various cases, which is defined by,

$$
\Delta q \Delta p \equiv \sqrt{ }\left\langle(q-\langle q\rangle)^{2}\right\rangle \sqrt{ }\left\langle(p-\langle p\rangle)^{2}\right\rangle,
$$

where $\langle\ldots\rangle$ denotes quantum mechanical average. The uncertainty product can be used as a measure of quantum fluctuation [34]. The initial value is $\Delta q \Delta p=\hbar / 2(=0.5)$ for the Gaussian wave packet. It is found that in the case $M=2$ the increase of the perturbation strength does not break the coherent oscillation and enhances the frequency of the time-dependence of the uncertainty product. For the relatively large $\epsilon$ in $M=10, \Delta q \Delta p$ increases until the wave packet is relaxed in the space, and it can not return to Gaussian wave packet anymore. For the larger time scale, it fluctuates around the corresponding certain level. We can expect that the structure of the time dependence well corresponds to the behavior of the transition probability $P_{L}(t)$ in Fig. 1 .

\section{D. stochastically perturbed case}

It will be instructive to compare the above irregular motion under the polychromatic perturbation with the stochastically perturbed one. We recall that the stochastic perturbation, composed of the infinite number of the frequency components $(M \rightarrow \infty)$ with absolute continuous spectrum, can break the coherent dynamics. Indeed, if the time dependence of the potential comes up with the stochastic fluctuation as $\left\langle\left(A\left(t_{1}\right)-a\right)\left(A\left(t_{2}\right)-a\right)\right\rangle_{e n} \propto$ $T_{B} \delta\left(t_{2}-t_{1}\right)$, where $\langle\ldots\rangle_{e n}$ and $T_{B}$ denote ensemble average and the temperature respectively, the stochastic perturbation partially models a heat bath coupled with the system 35]. Then the number of the frequency component corresponds to the number of degrees of freedom coupled with the double-well system. The $P_{L}(t)$ for the stochastic perturbation is shown in Fig. 6(a). The stochastic perturbation can be achieved numerically by replacing $A(t)-a$ in the Eq.(2) by random number and we use uniform random number which is normalized so that the power of the perturbation is the same order to 
one of the polychromatic case. In the limit of large $M$ the motion under the polychromatic perturbation tends to approach the one driven by the stochastic perturbation provided with the same perturbation strengths $\epsilon$.

Figure 7(b) shows the uncertainty product $\Delta q \Delta p$ for the stochastically perturbed cases. It is found that the time-dependence of the uncertainty product in the stochastically perturbed case behaves similarly to the polychromatically perturbed ones for the relatively small $\epsilon(\sim 0.1)$. On the other hand, for the relatively larger $\epsilon(=0.4)$ the time-dependence shows quite different behavior. While $\Delta q \Delta p$ grows linearly with time in the stochastically perturbed case, in the polychromatically perturbed cases the growth of $\Delta q \Delta p$ saturates at a certain level. The linear growth of $\Delta q \Delta p$ shows that the external stochasticity breaks the quantum interference in the internal dynamics. The growth of $\Delta q \Delta p$ is strongly related to the growth of the energy of the packet [26]. In the polychromatically perturbed cases the energy growth saturates at certain level due to quantum interference. On the other hand, in the case the energy grows unboundedly, the activation transition becomes much more dominant than the tunneling transition when the wave packet transfers the opposite well. The details concerning relation between the stochastic resonance [35] and suppression of the energy growth will be given elsewhere 26.

Note that the polychromatic perturbation can be identified with a white noise (or a colored noise if the frequencies are distributed over a finite band width) only in the limit of $M \rightarrow \infty$, while the stochastic perturbation can model a heat bath that breaks the quantum interference of the system. A similar phenomenon by the different property of the perturbation has been observed as "dynamical localization" and the "noise-assisted mixing" of the quantum state in the momentum space in the quantum kicked rotor model [36].

\section{E. phase space portrait}

Finally, in order to see effect of quantum fluctuation, we compare the quantum states with the classical and semiquantal motions in the phase space for some cases. The semiquantal equation of motion is given by generalized Hamilton-like equations as,

$$
\begin{aligned}
& \frac{d q}{d t}=p, \\
& \frac{d p}{d t}=-q^{3}+A(t) q-3 \hbar \phi q, \\
& \frac{d \phi}{d t}=4 \phi \pi, \\
& \frac{d \pi}{d t}=\frac{1}{8 \phi^{2}}-2 \pi^{2}-\frac{3}{4} q^{2}-\frac{1}{4} A(t)-\frac{3}{2} \hbar \phi
\end{aligned}
$$

where the canonical conjugate pair $(\phi, \pi)$ is defined by the quantum fluctuation $\Delta q$ and $\Delta p$ as, $\Delta q^{2}=\hbar \phi, \Delta p^{2}=$
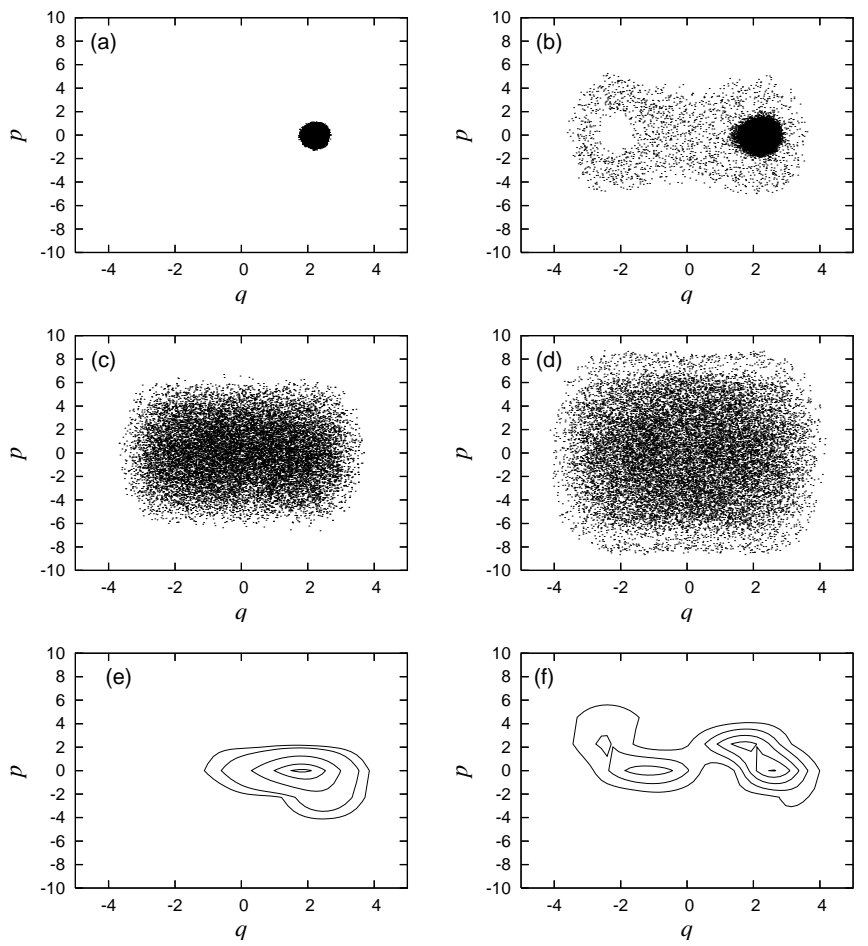

FIG. 8: Classical ((a), (b)) and semiquantal ((c), (d)) stroboscopic phase space portrait of the polychromatically perturbed double-well system with the same initial conditions $\left(q_{0}, p_{0}\right)$ and the same parameters at $t=2 \pi n / \Omega_{1}, n=1,2, \ldots$. The selection of initial condition of the fluctuation follows the minimum uncertainty. Contour plots ((e), (f)) of the Hushimi functions for the corresponding the quantum state at $t=8.7 \times 10^{3}$. Contour lines in the panel (e) at the values $0.01,0.02,0.05,0.08$ and 0.1 , in the panel (f) at 0.01, 0.02, $0.03,0.04$ and 0.05. $M=7$ and $\epsilon=0.4$ for (a), (c) and (e). $M=7$ and $\epsilon=0.8$ for (b), (d) and (f).

$\hbar\left(\frac{1}{4 \phi}+4 \pi^{2} \phi\right)$. For more details consult [4]. It is directly observed that the quantum tunneling phenomenon enhances chaotic motion in comparing to the classical and semiquantal trajectories. In Fig.8(a) and (b) Poincaré surface of section of the classical trajectories in the phase plane at $M=7$ are shown. The stroboscopic plots are taken at $t=2 \pi n / \Omega_{1}(n=1,2, \ldots)$, due to non timeperiodic structure of the Hamiltonian. In the relatively small perturbation strength $\epsilon=0.4$, the trajectories stay the single well, and are stable even for the long-time evolution. Figure 7(c) and (d) show the Poincaré section of the semiquantal trajectories for a polychromatically perturbed double-well system with $M=7$ (the stroboscopic plots are taken at $t=2 \pi n / \Omega_{1}$ again). The semiquantal trajectories for the squeezed quantum coherent state can be obtained by an effective action which includes partial quantum fluctuation to all order in $\hbar[3]$. It can be seen that in comparing with ones of classical dynamics the trajectories in the semiquantal dynamics spreads into the opposite well even for the small $\epsilon$. This corresponds to the quantum tunneling phenomenon through the semi- 
quantal dynamics. Apparently, the partial quantum fluctuation in the semiquantal approximation enhances the the chaotic behavior. Notice that the semiquantal picture breaks down for the irregular quantum states because the centroid motion becomes irrelevant.

In Fig.8(e) and (f) the corresponding coherent state representation for the quantum states are shown. It is directly seen that the wave packet spreads over the twowells and the shape is not symmetric. Once the wave packet incoherently spreads over the space, it can not return to the initial state anymore. We have confirmed that in a case without separatrix (single-well), namely the case that $a$ in Eq. (2) is replaced by $-a$, in the classical phase space the coherent oscillations have remained against the relatively large $M$ and/or $\epsilon$. It follows that the full quantum interference suppresses the chaotic behavior as seen in the semiquantual trajectories.

\section{SUMMARY AND DISCUSSION}

We numerically investigated influence of a polychromatic perturbation on wave packet dynamics in onedimensional double-well potential. The calculated physical quantities are the transition rate $P_{L}(t)$, the timefluctuation $\Delta P_{L}$, uncertainty product $\Delta q \Delta p$ and phase space portrait. The results we obtained in the present investigation are summarized as follows.

(1) We classified the motions in the parameter space spanned by the amplitude and the number of frequency components of the oscillatory driving force, i.e. coherent motions and irregular motions. The critical value $\epsilon_{c}(M)$ which divides the behavior of $P_{L}(t)$ into regular and irregular motions depends on the number of the frequency component $M$.

(2) Within the regular motion range, the period of the tunneling oscillation is reduced with increase of the number of colors and/or strength of the perturbation. It could be explained by the increase of the instanton tunneling rate due to appearance of the stochastic layer near separatrix [28]. In this parameter regime the perturbed instanton picture is one of expression for chaos-assisted tunneling 32] and chaos-assisted ionization picture reported for some quantum chaos systems 33.

(3) In the irregular motion in the polychromatically perturbed cases, the growth of $\Delta q \Delta p$ initially increases and saturates at certain level due to quantum interference. On the other hand, in the stochastically perturbed case the uncertainty product grows unboundedly because the external stochasticity breaks the quantum interference in the internal dynamics. The growth of $\Delta q \Delta p$ is strongly related to the growth of the energy of the wave packet.

(4) It is expected that the quantum fluctuation are always large for the classically chaotic trajectories compared to the regular ones. This implies that the quantum corrections to the evolution of the phase space fluctuation become more dominant for classically chaotic trajec- tories.

(5) In the semiquantal approximation the partial quantum fluctuation enhances the chaotic behavior, and simultaneously the chaos enhances the tunneling and decoherence of the wave packet. The quantum fluctuation observed in the semiquantal picture is suppressed by interference effect in the fully quantum motion. The semiquantal picture can not apply to the chaos-induced delocalized states.

Furthermore, in the appendices, we gave classical phase space portraits in the polychromatically perturbed double-well system and a simple explanation for the perturbed instanton tunneling picture for the reduction of the tunneling period in the coherent motion regime.

Although we have dealt with quantum dynamics of wave packet with paying attention to existence of the energetic barrier, we can expect that the similar phenomena would appear by dynamical barrier in the system. The details will be given elsewhere 26].

\section{APPENDIX A: EFFECT OF POLYCHROMATIC PERTURBATION ON CLASSICAL PHASE SPACE PORTRAITS}

We show classical stroboscopic phase space portrait in this appendix with paying an attention to the effect of polychromatic perturbation on the chaotic behavior. In the classical dynamics, such a system shows chaotic behavior by the oscillatory force $A(t)[24,26]$. The Newton's equation of the motion is

$$
\frac{d^{2} q}{d t^{2}}-A(t) q+q^{3}=0 .
$$

Note that in the monochromatically perturbed case $(M=1, A(t)=a-\epsilon \sin \Omega t)$, the equation is known as nonlinear Mathieu equation which can be derived from surface acoustic wave in piezoelectric solid [37] and nanomechanical amplifier in micronscale devices [38].

\section{Classical phase space potraits}

In Fig. A.1, we show the change of the classical stroboscopic phase space portrait changing the perturbation parameters. Increasing the perturbation strength $\epsilon$ destroys the separatrix and forms a chaotic layer in the vicinity of the separatrix. Needless to say, the phenomena have been observed even in the monochromatically perturbed cases 24]. In the polychromatically perturbed cases $(M>1)$ the smaller the strength $\epsilon$ can generate chaotic behavior of the classical trajectories the larger $M$ is [26]. It should be emphasized that in the polychromatically perturbed cases the width of the chaotic layer grows faster than the monochromatically perturbed case 
as the perturbation strength increases. As a result, the increase of the color contributes the increase of the width of the stochastic layer in the polychromatically perturbed cases.
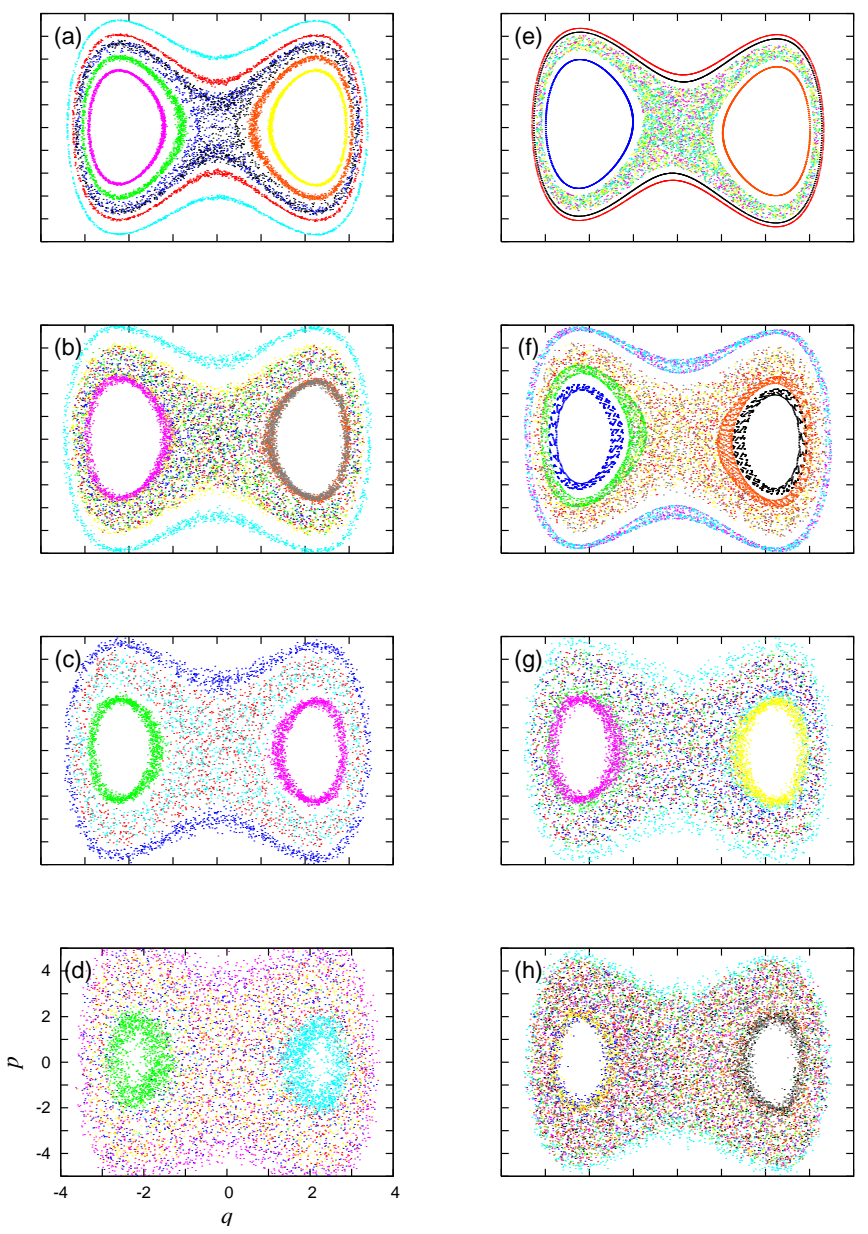

FIG. 9: Stroboscopic classical phase space portraits of the polychromatically perturbed double-well system. Cross sections of $(p, q)$ space are plotted at $2 \pi n / \Omega_{1}, n=1,2,3, \ldots$. The $\epsilon$-dependence for $M=5$ is shown in (a) $\epsilon=0.1$, (b) $\epsilon=0.3$, (c) $\epsilon=0.4$ and $(\mathrm{d}) \epsilon=0.8$. The $M$-dependence for $\epsilon=0.5$ is shown in (e) $M=1$, (f) $M=2$, (g) $M=5$ and (h) $M=8$.

Here, we use the increasing rate of infinitesimal displacement along the classical trajectory for the extent of chaotic behavior as a finite-time Lyapunov exponent $\lambda_{c l}^{\max }$. We prepare various initial points in the phase space, and conveniently adapt a trajectory with maximal increasing rate among the ensemble within the finite-time interval as the finite-time Lyapunov exponent. Note that an exact Lyapunov exponent should be defined for the long-time limit. However, the roughly estimated Lyapunov exponent is also useful to observe the classical-quantum correspondence. Figure A.2 shows the $\epsilon$-dependence of classical Lyapunov exponents for various cases estimated by the numerical data of the classical trajectories.

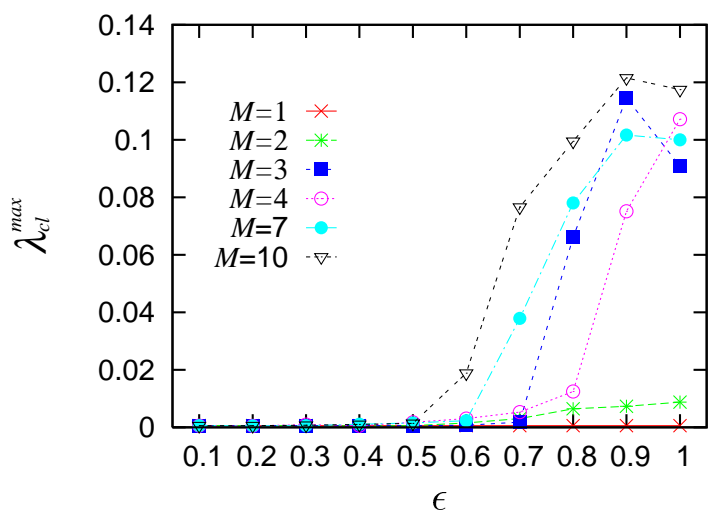

FIG. 10: Maximal Lyapunov exponents $\lambda_{c l}^{\max }$ estimated by some classical trajectories within the finite-time $t \in[0, T]$, where $T$ is tunneling time given inSubsect.3.1.

We can roughly observe a transition from motion of KAM system $\left(\lambda_{c l}^{\max } \sim 0\right)$ to chaotic motion $\left(\lambda_{c l}^{\max }>0\right)$ as the perturbation strength increases. The increasing of the number of color $M$ reduces the value of the critical perturbation strength $\epsilon_{c}^{c l}$ of a transition from a motion of KAM system to fully chaotic motion. Roughly speaking, a transition of the classical dynamics corresponds to the transition from coherent motion to irregular one in the quantum dynamics. We expect that the transition observed in Sect.3 will corresponds to quantum signatures of the KAM transition from the regular to chaotic dynamics.

\section{Some considerations to the effect of polychromatic perturbation}

In this subsection, we conceptually consider a role of the polychromatic perturbation different from monochromatic one. Note that the change of the number $M$ of colors also changes the qualitative nature of the underlying dynamics because $M$ corresponds to the effective number of DOF under some conditions [25, 41]. In the our model, when the number of DOF of the total system is more than four, i.e. $M \geq 3$, the classical trajectories can diffuse along the stochastic layers of many resonances that cover the whole phase space if the trajectory starts in the vicinity of a nonlinear resonance. The number of resonances increases rapidly with DOF, changing the characteristic of population transfer from bounded to diffusive. Such a global instability is known as Arnold diffusion in nonlinear Hamiltonian system with many DOF [39]. The effect of Arnold diffusion in quantum system is not trivial and the study just has started recently [40]. The more detail is out of scope of this paper.

Moreover, we can regard the time-dependent model of Eq.(1) as nonautonomous approximation for an autonomous model, consisting of the double-well system coupled finite number $M$ of harmonic oscillators with 
the incommensurate frequencies $\left\{\Omega_{j}, j=1,1, \ldots, M\right\}$. It is worth noting that the linear oscillators can be identified with a highly excited quantum harmonic oscillators, which all phonon modes are excited around Fock states with large quantum numbers. Then the above model can be regarded as a double-well system coupled with $M$ phonon modes. Without the interaction with the phonon modes the Gaussian wavepacket remains the coherent motion. Then the number of DOF of total system is $1+M$ and the number of the frequency components $M$ corresponds to the that of the highly excited quantum harmonic oscillators. The detail of the correspondence is given in Ref. [25].

In quantum chaotic system with finite and many DOF we expect occurrence of a dissipative behavior. For example, we consider simulated light absorption by coupling a system in the ground state with radiation field. Then stationary one-way energy transport from photon source to the system can be interpreted as occurrence of quantum irreversibility in the total system. Such a irreversibility is called chaos-induced dissipation in quantum system with more than two DOF [41]. In this sense, we can expect occurrence of the one-way transport phenomenon in the delocalized state in the irregular motion phase if it couples with the other DOF in the ground state as seen in Ref. 25].

\section{APPENDIX B: PERTURBED INSTANTON TUNNELING}

In this appendix, we consider the reduction of the tunneling period as the perturbation strength increases in the coherent oscilation regime $\epsilon<\epsilon_{c}$, based on a perturbed instanton tunneling. In a double-well system with dipole-type interaction, $q \sin (\Omega t)$, the energitical barrier tunneling between the symmetric double-well can be explained by a three-state model or chaos-assisted tunneling (CAT) 30, 31, 32, 33. The three states that take part in the tunneling are a doublet of quasi-degenerate states with opposite parity, localized in the each well, and a third state localized in the chaotic layer around the separatrix.

However, note that less attention has been paid to tunneling in KAM system while chaotic dynamics has been modeled by multi-level Hamiltonian and random matrix model to describe the chaos-assisted tunneling. We give an expresion of the tunneling amplitude in "chaosassisted instanton tunneling" firstly proposed by Kuvshinov et al for a Hamiltonian system with time-periodic perturbation [28]. Let us consider only monochromatically perturbed case ( $M=1$ in Eq.(2), $\Omega=\Omega_{1}$ ) because the separatrix destruction mechanism by the timeperiodic perturbation has an universality although our system is different from their one. .

\section{A discrete mapping}

Indeed, trajectories in the neighborhood of the separatrix of the system are well reproduced by the whisker map of the system. Whisker map is a map of the energy change $I_{n}$ and phase change $\phi_{n}$ of a trajectory in the neighborhood of the separatrix for each of its motion during one period of the perturbation, i.e. action-angle variable. Moreover, if we linearize the whisker map which describes the behaviors of the trajectories in the neighborhood of the fixed point, we can obtain the following standard map,

$$
\left\{\begin{array}{l}
I_{n+1}=I_{n}-K(\epsilon, \Omega) \sin \phi_{n} \\
\phi_{n+1}=\phi_{n}+I_{n+1}
\end{array}\right.
$$

, where $K(\epsilon, \Omega)$ is a nonlinear parameter of local instability that the exact function form which is not essential for our purpose. $K$ increases with $\epsilon$, and $K \geq 1$ means that the dynamics of the system is locally unstable. A comparison has done between the whisker map and the strobe plots in the time continuous version by Yamaguchi [21]. The form of the mapping is convenient for the estimate of the width of the stochastic layer.

Kuvshivov et al has given the estimated parameter of local instability, width of stochastic layer, and correlator for perturbed instanton solution for the perturbed pendulum system [28].

\section{Action of the instanton tunneling}

The perturbation destroys separatrix of the unperturbed system and the stochastic layer appears. In the regular motion denoted by the circles in Fig.3, classical chaos can increase the rate of instanton tunneling due to appearance of the stochastic layer near separatrix of the unperturbed system. As a result the frequency of time-dependence $P_{L}(t)$ increases as the classical chaos becomes remarkable in the parameter regime. Note that the perturbed instanton tunneling picture disappears in the strongly perturbed regime due to the delocalization of wavepacket.

Here we give only relation between the width of the stochastic layer and the tunneling amplitude in terms of path integral in imaginary time $\tau$, found by Kuvshivov et al. Tunneling amplitude between the two wells in the perturbed system can be given by integration over energy of tunneling amplitude $A_{\text {tun }}$ in unperturbed system as,

$$
A_{\text {tun }}=\int_{0}^{\Delta H} d E \int_{q(\tau)=-q_{0}}^{q(\tau)=q_{0}} D[q(\tau, E)] \exp \{-S[q(\tau, \mathbb{E B B}]\})
$$

where $S[q(\tau, E)]$ denotes the Euclidian action. $\Delta H \equiv$ $2\left|H_{s}-H_{b}\right|$ denotes the width of stochastic layer, where $H_{s}$ and $H_{b}$ are the energy of the unperturbed system on the separatrix and on the bound of stochastic layer, respectively. $q(\tau, E)$ is classical solution of Euclidian equation of motion. The contribution of the chaotic instanton 
solution are taken into account by means of integration over $E$ which is energy of the instanton.

The perturbed instanton solutions correspond to the motions in vicinity of the separatrix inside the layer. The only manifestation of the perturbation in this approximation is the appearance of a number of additional solutions of the Euclidian equation of motion with energy close to the energy of the unperturbed one-instanton solution inside the stochastic layer. Accordingly, we can expect that the appearance of the stochastic layer enhances the tunneling rate as reported in the other systems [28]. However, we have to have in mind that the result is obtained in the first order on coupling constant $\epsilon$ of the time-periodic perturbation and does not take into account the structure of stochastic layer. The approximation is valid if the layer is narrow by neglecting the higher order resonances in the phase space. For the more details of the perturbed instanton see Ref. [28]. The increasing of the tunneling amplitude is directly related to energy splitting $\Delta E_{01}$ between the quasi-degenerate ground Floquet states.

As seen in Fig.A.1, the increasing of number of color $M$ can enhance the width of the stochastic layer with the perturbation strength being kept at a constant value. The theoretical explanation for the reduction of the tunneling period with the number of color is open for further study.

We expect that in the double-well system under the polychromatic perturbation this numerical study will be useful for the analytical derivation of "reduction of tunneling period" and "critical strength of a transition from localized to delocalized behavior of wavepacket" by extension of the monochromatically perturbed case. The chaos assisted instanton theory might be applicable if we will exactly estimate the width of the stochastic layer in the system under the polychromatic perturbation.
[1] M. Gutzwiller, Chaos in Classical and Quantum Mechanics (Springer, Berlin, 1990).

[2] B. Hu, B. Li, J. Liu and J.L. Zhou, Phys. Rev. E 58, 1743(1998).

[3] A.K. Pattanayak and W.C. Schieve, Phys. Rev. Lett. 72, 2855(1994) ;Y.H. Wong, X.T. Mo and W.M. Zhang, Chin. J. Phys., 37, 466(1999).

[4] W.M. Zhang and D.H. Feng, Phys. Rep. 252, 1(1995).

[5] M. Razavy, Quantum Theory of Tunneling (World Scientific, 2003).

[6] Tunneling in Complex Systems (World Scientific, 1998), edited by $\mathrm{S}$. Tomsovic.

[7] A. Shudo and K.S. Ikeda, Phyasica D 115, 234(1998); T. Onishi, A. Shudo, K. S. Ikeda, K. Takahashi, Phys. Rev. E 68, 056211(2003).

[8] K. Takatsuka, H. Ushiyama and A.I. Ushiyama, Phys. Rep. 322, 347(1999).

[9] O. Brodier, P. Schlagheck and D. Ullmo, Ann. Phys. 300, 88(2002).

[10] W.A. Lin and L.E. Ballentine, Phys. Rev. Lett. 65, 2927(1990); Phys. Rev. A 45, 3637(1992).

[11] F. Grossmann, T. Dittrich, P. Jung and P. Hänggi, Phys. Rev. Lett. 67, 516(1991); M. Grifoni and P. Hänggi, Phys. Rep. 304, 229(1998).

[12] U. Weiss, Quantum Dissipative Systems, second edition (World Scientific, 1999).

[13] A.O. Caldeira and A.J. Leggett, Phys. Rev. Lett. 46, 211(1981).

[14] T. Dittrich and R. Graham, Z. Phys. B 62, 515(1986); Ann. Phys. 200, 363(1990).

[15] D. Cohen, Phys. Rev. Lett. 82, 4951(1999).

[16] W.H. Zurek, Phys. Rev. D 24, 1516(1981); W.H. Zurek and J.P. Paz, Phys Rev. Lett. 72, 2508(1994); S. Habib, et al., Phys. Rev. Lett. 88, 040402(2002).

[17] D. Cohen, Phys Rev. Lett. 78, 2878(1997).

[18] A.R. Kolovsky, S. Miyazaki, and R. Graham, Phys. Rev. E 49, 70(1994).

[19] G. Casati, I. Guarneri and D.L. Shepelyansky, Phys. Rev. Lett. 62, 345(1989).

[20] F. Borgonovi and D.L. Shepelyansky, Physica D 109,
24(1997).

[21] Y. Yamaguchi, Phys. Lett. A 109, 191(1985).

[22] J. Guckenheimer and P. Holmes, Nonlinear Oscillations, Dynamical Systems, and Bifurcations of Vector fields (Springer-verlag, New York, 1983).

[23] We have confirmed that result in the polychromatically perturbed Duffing-like model was qualitatively same as the present one.

[24] A. Igarashi and H. Yamada, Chem. Phys. 309, 95(2005).

[25] H. Yamada and K.S. Ikeda, Phys. Rev. E 59, 5214(1999); ibid, 65, 046211(2002).

[26] A. Igarashi and H. Yamada, in preparation.

[27] For example, T. Prosen, Phys. Rev. E 65, 036208(2002).

[28] V.I. Kuvshinov, A.V. Kuzmin, and R.G. Shulyakovsky, Phys. Rev. E 67, 015201(2003); Acta Phys. Polon. B 33, 1721(2002).

[29] M. Holthaus, Phys. Rev. Lett. 69, 1596(1992).

[30] S. Tomsovic and D. Ullmo, Phys. Rev. E 50, 145(1994).

[31] S.Kohler, R. Utermann, P. Hänggi and T. Dittrich, Phys. Rev. E 58, 7219(1998).

[32] L. Bonci et al., Phys. Rev. E 58, 5689(1998); D.A. Steck, et al., Phys. Rev. Lett. 88, 120406(2002).

[33] J. Zakrzewski, et al., Phys. Rev. E 57, 1458(1998); K. Sacha, et al., Ann. Phys. 283, 141(2000).

[34] It should be noted that the uncertainty product is not always good measure of quantum fluctuation because it does not correspond to the real area (or volume) of phase space. However, we would like to pay attention to the initial growth and the mean value during the time-evolution process instead of the detail of the definition of the exact quantum fluctuation in the dynamics. Practically, the $\Delta p \Delta q$ remains small thorough the regular instanton-like motion, on the other hand, it shows sharp increase and the large value remains for the strongly chaotic cases. See papers, S. Chaudhuri, G. Gangopadhyay and D.S.Ray, Phys. Lett. A 216, 53(1996); P. K. Chattaraj, B. Maiti, and S. Sengupta, Int. J. Quant. Chem. 100, 254(2004). As a result the classical chaos enhances quantum fluctuation in the restricted sense.

[35] L. Gammaitoni, P. Hänggi, P. Jung and F. Marchesoni, 
Rev. Mod. Phys. 70, 223(1998).

[36] S. Adachi, M. Toda and K. Ikeda, Phys. Rev. Lett. 61, 655(1988).

[37] H. Konno, J. Phys. Soc. Jpn. 59, 3989(1990).

[38] D.A. Harrington and M.L. Roukes, preprint(2002).

[39] B.V. Chirikov, Phys. Rep. 52, 263(1979); C.C. Martens,
M.J. Davis and G.S. Ezra, Chem. Phys. Lett. 142 , 519(1987).

[40] For example, see, V.Ya.Demikhovskii, F.M.Izrailev, and A.I.Malyshev, Phys. Rev. Lett. 88, 154101(2002).

[41] K. Ikeda, Ann. Phys. (N.Y.) 227, 1(1993). 